DOI: $10.1002 /(($ please add manuscript number))

Article type: Communication

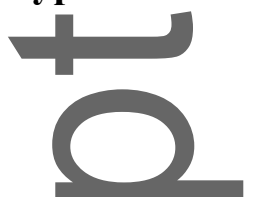

\title{
Sodium-Doped Tin Sulfide Single Crystal: A Nontoxic Earth-Abundant Material with High Thermoelectric Performance
}

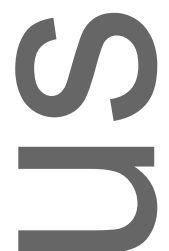

Hong Wu, Xu Lu, Guoyu Wang, Kunling Peng, Hang Chi, Bin Zhang, Yongjin Chen, Chengjun Li, Yanci Yan, Lijie Guo, Ctirad Uher, Xiaoyuan Zhou*, Xiaodong Han*

H. Wu, K. L. Peng, C. J. Li, L. J. Guo, Y. C. Yan, Prof. X. Lu, Prof. X.Y Zhou

College of Physics, Chongqing University, Chongqing 401331, P. R. China

Email: xiaoytran2013@cqu.edu.cn

Y. J. Chen, Prof. X.D. Han

Beijing Key Laboratory of Microstructure and Property of Advanced Materials, Beijing University of Technology, Beijing 100024, P. R. China

Email: xdhan@bjut.edu.cn

Dr. B. Zhang, Prof. X.Y. Zhou

Analytical and Testing Center of Chongqing University, Chongqing 401331, P. R. China

Dr. H. Chi, Prof. C. Uher

This is the ant - manuscript accepted for publication and has undergone full peer review but has not been thioh tl copyediting, typesetting, pagination and proofreading process, which may lead to differences betm this version and the Version of Record. Please cite this article as doi: 10.1002/aenm.201800087.

This article is protected by copyright. All rights reserved. 
Department of Physics, University of Michigan, Ann Arbor, MI 48109, USA

H. Wu, Prof. G. Y. Wang

Chongqing Institute of Green and Intelligent Technology, Chinese Academy of Sciences Chongqing 400714, P. R. China and University of Chinese Academy of Sciences, Beijing, 100044, P. R. China

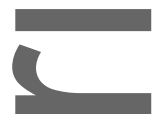

Keywords: thermoelectric, SnS single crystal, electronic structure, SPB model

Abstract: Lead-free tin sulfide ( $\mathrm{SnS})$, with an analogous structure to SnSe, has attracted increasingattentions because of its theoretically predicted high thermoelectric performance. In practice, however, polycrystalline $\mathrm{SnS}$ performs rather poorly as a result of its low power factor. In this work, we have synthesized bulk sodium ( $\mathrm{Na}$ )-doped $\mathrm{SnS}$ single crystals using a modified Bridgman method and conducted a detailed transport evaluation. The highest $z T$ value of $\sim 1.1$ was reached at $870 \mathrm{~K}$ in a 2 at.\% $\mathrm{Na}$-doped $\mathrm{SnS}$ single crystal along the $b$-axis direction, in which high power factors $\left(2.0 \mathrm{~mW} \mathrm{~m}^{-1} \mathrm{~K}^{-2}\right.$ at room temperature $)$ were realized. We attributed these high power factors to the high mobility associated with the single crystalline nature of the samples as well as to the enhanced carrier concentration achieved through Na doping. We used an effective single parabolic band model coupled with first-principles calculations to provide theoretical insight into the electronic transport properties. Our work demonstrates that SnS-based single crystals composed of earth-abundant, 10w-cost and nontoxic chemical elements can exhibit high thermoelectric performance and thus hold potential for application in the area of waste heat recovery.

\section{Introduction}


To alleviate worldwide energy shortages and environmental issues caused by the excessive use of fossil fuel-generated energy, the development of green energy technology has become imperative. One solution has been to use thermoelectric (TE) devices, which can directly convert waste heat into electricity without generating any additional pollution. ${ }^{[1-3]}$ In general, TE materials that contain inexpensive earth-abundant, and nontoxic elements are the most interesting applications. ${ }^{[4,5]}$ The conversion efficiency for a TE material is determined by the dimensionless figure of merit $(z T)$, defined as $z T=S^{2} \rho^{-1} \kappa^{-1} T$, where $S$ is the Seebeck coefficient, $\rho$ is the electrical resistivity, $T$ is the absolute temperature and $\kappa$ is the total thermal conductivity consisting of the lattice part $\left(\kappa_{\mathrm{L}}\right)$ and the electronic part $\left(\kappa_{\mathrm{e}}\right) .{ }^{[6]}$ Some of these parameters $\left(S, \rho\right.$, and $\left.\kappa_{\mathrm{e}}\right)$, however, are mutually interdependent and strongly coupled by way of the carrier-concentration, making it difficult to attain high TE performance $(z T)$ without making some compromise. Fortunately, in the past decade, several general strategies have been developed to achieve high $z T$ in various materials. ${ }^{[7,8]}$ Lowering the lattice thermal conductivity (א,, which is relatively independent of other parameters, is a comparatively easy method. Other The well-documented methods for enhancing phonon scattering include nanostructuring, ${ }^{[9-11]}$ dislocations, ${ }^{[12,13]}$ point defects ${ }^{[14-17]}$ and strong lattice anharmonicity. ${ }^{[18-20]}$ Additional strategies to boost the power factor $\left(S^{2} \rho^{-1}\right)$ include carrier concentration optimization, ${ }^{[21,22]}$ band convergence, ${ }^{[23,24]}$ and creating band resonance levels. ${ }^{[25]}$

Typically, practical TE materials are polycrystalline structures with grain boundaries that help to maintain a low lattice thermal conductivity but inevitably also degrade the charge carrier mobility. Recently, bulk $\mathrm{SnSe}^{[26-30]}$ and $\mathrm{In}_{4} \mathrm{Se}_{3}{ }^{[31]}$ single crystals have attracted increasing amounts of attention. The layered structure and strong lattice anharmonicity of 
these materials cause ultralow lattice thermal conductivity, independent of grain boundary phonon scattering. Therefore, such single crystalline compounds are expected to yield superior performance as both high mobility and low lattice thermal conductivity can be maintained simultaneously. For instance, the ultrahigh $z T$ of $\sim 2.6$ at $923 \mathrm{~K}$ for $\mathrm{SnSe}$ single crystals along the $b$-axis has been achieved. ${ }^{[26]}$

The analogous crystal structure of $\mathrm{SnS}$ to SnSe has suggested that potentially it also should have ultralow lattice thermal conductivity. Indeed, the first-principles calculations predict a high Seebeck coefficient $(S)$ and low thermal conductivity $(\kappa)$ for SnS. ${ }^{[32,33]}$ The recent study using polycrystalline SnS samples was rather disappointing in its outcome as it resulted in a relatively low $z T$ value of 0.65 at $850 \mathrm{~K},{ }^{[34]}$ suffering chiefly from the low power factors of the polycrystalline samples. The prospect of achieving significantly higher power factors with single crystal samples of $\mathrm{SnS}$ and the lack of information regarding their transport properties have motivated us to perform a comprehensive study of the TE performance of single-crystalline forms of $\mathrm{SnS}$.

In this work, we grew bulk SnS single crystals using a modified Bridgman method. The denotation of three principle crystallography directions is shown in Figure S1(a). Like other single crystal, the transport properties of SnS exhibit strong anisotropy, which will be demonstrated in the following section. We focus on the favorable direction ( $b$ direction) first and cover the details of anisotropy in the last section. The hole concentration is significantly improved by sodium $(\mathrm{Na})$ doping on the sites of tin $(\mathrm{Sn})$. Meanwhile, the carrier mobility is high compared with SnS polycrystalline samples because of the lack of grain boundary scattering. As a result, we achieved a high power factor $\left(2.0 \mathrm{~mW} \mathrm{~m}^{-1} \mathrm{~K}^{-2}\right)$ at room temperature. We maintained the power factor above $0.75 \mathrm{~mW} \mathrm{~m}^{-1} \mathrm{~K}^{-2}$ at elevated 
temperatures for the 2 at.\% Na-doped $\mathrm{SnS}$ along the $b$-axis direction, which enabled a high $z T$ value of $\sim 1.1$ at $870 \mathrm{~K}$. Furthermore, an analysis based on the effective single parabolic band (SPB) model coupled with first-principles calculations proved that the high power factor primarily resulted from the secondary heavy valence band contributing to transport.

\section{Results and discussion}

Crystal structure characterizations: Figure 1(a) shows X-ray diffraction (XRD) of $\mathrm{Sn}_{1-x} \mathrm{Na}_{x} \mathrm{~S}(x=0,0.001,0.01,0.02,0.03,0.04)$ single crystals obtained on the $(100)$ cleavage surface, similar to SnSe single crystals. ${ }^{[26,27]}$ Additionally, we collected powder XRD patterns, which are shown in Figure S1(b) for phase identification and crystalline parameter determination. All major reflection peaks can be indexed to those of PDF\#014-0620, indicating the crystal structure of the $\mathrm{Sn}_{1-x} \mathrm{Na}_{x} \mathrm{~S}$ single crystals belongs to the Pnma space group and no secondary phases are present. Results of Rietveld refinement for undoped and Na-doped SnS crystals are shown in Table S1. We performed scanning transmission electron microscopy high-angle annular dark field (STEM-HAADF) and energy-dispersive X-ray spectrum (EDS) mapping on the $\mathrm{Sn}_{0.98} \mathrm{Na}_{0.02} \mathrm{~S}$ crystal to obtain its detailed structural analysis. As illustrated by the structure model (Figure 1(b)), the double Sn-S planes are linked by the van der Waals force along the [100] direction, which was confirmed by the HAADF image (Figure 1(c)). Thus, it is easy to cleave SnS single crystals (both doped and undoped) along the (100) planes, as illustrated in Figure 1(b). The HAADF image and the corresponding EDS mapping with atomic-scale resolvable structural identification are shown in Figures 1(d)-1(g). As expected, Sn and S atoms are located on different columns of the [001] projection. In the HAADF images, Sn columns appear as 
bright dots because of their large atomic number (50), whereas S columns are dimmer, as further confirmed by EDS mappings. A schematic of the atomic structure is attached at the bottom right corner of the HAADF image (Figure 1(d)) and at the bottom right corner of the combined EDS mapping (Figure 1(g)). Finally, note that because of the small content and weak EDX signals, we do not present the EDS mapping graphic of $\mathrm{Na}$.

Electronic transport properties: To better understand the physical nature of electronic transport properties, we calculated the band structure of SnS (Pnma space group) along the high symmetry directions. As shown in Figure 2(a), the calculated band gap of $\mathrm{SnS}$ is $\sim 0.89$ $\mathrm{eV}$, which agrees with the results of previous calculations ${ }^{[39]}$ and is smaller than that which we obtained experimentally from optical measurements $(\sim 1.1 \mathrm{eV}$; see Figure S6), because of the intrinsic underestimation of band gap sizes in the density functional theory (DFT) calculations. Weassumed that the slight $(\sim 10 \%)$ underestimation of the band gap would not affect the band edge morphology, as clarified in previous reports for SnSe. ${ }^{[27]}$ As shown in Figure 2(a), the valence band maximum was situated at GZ1 $(0.0,0.0,0.448)$ along the $\Gamma-Z$ symmetry direction, whereas the position of the second valence band maximum was situated at GA1 $(0.466,0.0,0.466)$ along the $\Gamma$-A direction. The energy difference between these two valence band extrema was $\sim 0.055 \mathrm{eV}$ (see Table 1), which was comparable to that of SnSe $(\sim 0.057 \mathrm{eV}) \cdot{ }^{[27]}$ Consequently, we expected that the second band could play an important role in the electronic transport when the structure was sufficiently doped. To clarify variations of the Fermi level with respect to the Na-doping content in SnS, which determined the number of carrier poekets participating in the carrier transport, we applied the BoltzTrap code ${ }^{[40,41]}$ to calculate the corresponding carrier concentration when the Fermi level reached different extreme points. Figure 2(a) shows that the Fermi level pushed down into the second carrier 
pockets (GA1) when the carrier concentration reached $\sim 5.0 \times 10^{19} \mathrm{~cm}^{-3}$ at $300 \mathrm{~K}$, suggesting that multiple carrier pockets contributed to the carrier transport, which is beneficial for the enhancement of the power factor. In addition, the density of states (DOS) for pristine $\mathrm{SnS}$ is displayed in Figure 2(b), which clearly shows that the DOS near the valence band edge principally originated from the contribution of $p$-orbitals of $\mathrm{S}$ atoms.

As listed in Table 1, we calculated the band effective masses at GZ1 and GA1; the method of calculations is provided in the supporting information. The result of calculations at each specific extreme point yielded anisotropic effective masses, with the effective mass along the $k_{x}$ direction being larger than the mass in the other two directions ( $k_{y}$ and $k_{z}$ ), because of the quasi-laminar and highly anisotropic structure of SnS. This was consistent with a previous report, ${ }^{[39]}$ in which the effective masses of the first band $\left(m^{*}{ }_{k x}=2.63 m_{e}, m_{k y}{ }_{k y}=0.32 m_{e}\right.$ and $\left.m_{k z}^{*}=0.20 m_{e}\right)$ and those of the second band $\left(m_{k x}=8.48 m_{e}, m_{k y}=0.29 m_{e}\right.$ and $m_{k z}=0.54$ $\left.m_{e}\right)$ were reported. Hence, the DOS effective mass of the second band $\left(\sim 3.063 m_{e}\right)$ was three times larger than that of the first band $\left(\sim 0.971 m_{e}\right)$, indicating a potentially enhanced Seebeck coefficient benefiting from the presence of such heavy band.

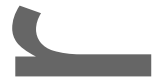

It is assumed that the band gap $(\sim 1.1 \mathrm{eV})$ is large enough for $\mathrm{SnS}$ to exhibit the parabolic character at the band edges. In addition, the Hall mobility decreased with increasing temperature, following the relationship of $\mu \sim T^{-1.5}$ (Figure 3(c)). This suggested that the charge carriers mainly were scattered by acoustic phonons. Thereby, we are able to use an equivalent SPB)model to make a Pisarenko plot and further illustrate the behavior of electronic transport properties (detailed calculations are presented in the supporting information). As shown in Figure 3(e), the experimental carrier concentration and the Seebeck coefficient fit well the Pisarenko line using the effective SPB model with different 
DOS effective masses. We found that the DOS effective mass guided by the navy dash line $\left(m^{*}=0.793 m_{e}\right)$ fit the undoped sample $\left(\sim 2.0 \times 10^{17} \mathrm{~cm}^{-3}\right)$, and the dependence of Na-doped single crystal samples could be fit with $m^{*}=1.41 m_{e}$ at $300 \mathrm{~K}$. By combining fitting with the DOS effective mass obtained from DFT calculations (see Table 1) and the shift of the Fermi level with different carrier concentration using the BoltzTrap code (Figure 2(a)), we speculated that the Fermi level would be above the valence band edge for the undoped sample, whereas the lower-lying heavy band began to contribute when the crystals were heavily doped with Na. To further validate this hypothesis, we performed fitting for experimental data at $500 \mathrm{~K}$ (Figure 3(e)). The DOS effective mass obtained from fitting slightly increased with temperature for the undoped sample. Furthermore, the Hall carrier concentration was consistent with the fitting result based on the SPB model, as listed in Table 2.

Figure 3 displays the temperature-dependent electronic properties of undoped and Na-doped SnS single crystals along the $b$-axis. The electrical resistivity $(\rho)$, Figure 3(a), of all crystals initially rose with the increasing temperature, showing a metallic behavior, reached a maximum, and then decreased. The temperature at which the resistivity reached its peak value shifted to higher temperatures as the carrier concentration increased. The eventual decrease in the resistivity was caused by the onset of intrinsic excitations. The magnitude of the electrical resistivity decreased sequentially with the increasing $\mathrm{Na}$ content (up to $2 \%$ ), obviously because of the boosted carrier concentration upon doping. For instance, the Hall carrier concentration at $300 \mathrm{~K}$ was significantly enhanced from $\sim 2.0 \times 10^{17} \mathrm{~cm}^{-3}$ for the undoped SnS crystal to $2.3 \times 10^{19} \mathrm{~cm}^{-3}$ for the $\mathrm{Na}_{0.02} \mathrm{Sn}_{0.98} \mathrm{~S}$ crystal; see Figure 3(b). Note, however, that in comparison to the effect of $\mathrm{Na}$ doping in SnSe, ${ }^{[27]}$ the doping efficiency of 
$\mathrm{Na}$ in $\mathrm{SnS}$ was lower, and this may have limited further enhancements in the TE performance of $\mathrm{SnS}$. The onset of intrinsic excitations is clearly seen in Figure 3(b) on the carrier concentration of pure SnS that underwent a rapid increase above $700 \mathrm{~K}$. As shown in Figure 3(c), the temperature-dependence of the Hall mobility, calculated from $\mu_{H}=\rho^{-1} e^{-1} n_{H}{ }^{-1}$, followed the $\mu \approx T^{-1.5}$ dependence, implying the dominance of acoustic phonon scattering. As expected, the Hall mobility for the undoped SnS crystal was higher than for the Na-doped SnS crystals over the whole temperature range. We attributed this higher mobility to alloy scattering because of the mass difference and the atomic radii difference between $\mathrm{Na}$ and $\mathrm{Sn}$. Compared with polycrystalline samples of $\operatorname{SnS}\left(\sim 10 \mathrm{~cm}^{2} \mathrm{~V}^{-1} \mathrm{~s}^{-1}\right)$, the carrier mobility in our single crystals was greatly improved, which undoubtedly was the consequence of strongly diminished charge scattering by grain boundaries.

The positive values of the Seebeck coefficient shown in Figure 3(d) indicated that all crystals were $p$-type semiconductors. As expected, the Seebeck coefficient clearly diminished as the Hallearrier concentration increased. At elevated temperatures, the Seebeck coefficient turned over and decreased. This, again, is a distinct signature of the onset of intrinsic excitations at which point the minority electrons start to compensate the majority holes. The turn over shifted to higher temperatures as the carrier concentration of holes increased. Figure 3(f) presents the power factor of all $\mathrm{SnS}$ crystals. We achieved the maximum power factor of $2.0 \mathrm{~mW} \mathrm{~m}^{-1} \mathrm{~K}^{-2}$ at room temperature in an $\mathrm{SnS}$ crystal doped with 2 at.\% Na. This value of the power factor was substantially higher than that of the pure $\mathrm{SnS}$ crystal $(0.19 \mathrm{~mW}$ $\mathrm{m}^{-1} \mathrm{~K}^{-2}$ ) and significantly exceeded the previously reported power factor of polycrystalline SnS samples. ${ }^{[34,35]}$ 
Thermal properties: Figures 4(a) and 4(b) display the temperature-dependent total and lattice thermal conductivity along the $b$-axis direction for all single crystal samples. The total thermal conductivity $(\kappa)$ of our crystals ranged from $2.36 \mathrm{~W} \mathrm{~m}^{-1} \mathrm{~K}^{-1}$ to $2.87 \mathrm{~W} \mathrm{~m}^{-1} \mathrm{~K}^{-1}$ at $320 \mathrm{~K}$, which was higher than that for the SnSe single crystal along the same direction. ${ }^{[30]}$ The total thermal conductivity of all SnS crystals decreased as the temperature increased. We attained the minimum value of $0.61 \mathrm{~W} \mathrm{~m}^{-1} \mathrm{~K}^{-1}$ for the $\mathrm{Na}_{0.02} \mathrm{Sn}_{0.98} \mathrm{~S}$ single crystal along the $b$-axis at about $870 \mathrm{~K}$. We obtained the lattice thermal conductivity $\left(\kappa_{L}\right)$ of all samples by subtracting the electronic contribution $\left(\kappa_{\mathrm{e}}\right)$ from the total thermal conductivity, $\left(\kappa_{L}=\kappa-\kappa_{\mathrm{e}}\right)$. The electronic thermal conductivity contribution $\left(\kappa_{\mathrm{e}}\right)$ was calculated according to the Wiedemann-Franz law $\left(\kappa_{\mathrm{e}}=L T \rho^{-1}\right)$. Here, $L$ is the Lorenz number, which is estimated based on the SPB model (see the supporting information). Comparing Figures 4(a) and 4(b), it follows that the electronic thermal conductivity of all $\mathrm{SnS}$ crystals represented a minor fraction of the oyerall thermal conductivity. The lattice thermal conductivity exhibited a sharp downward trend closely following the $T^{-1}$ law and indicating the dominance of phonon Umklapp scattering processes. We achieved the minimum lattice thermal conductivity of 0.54 $\mathrm{W} \mathrm{m}^{-1} \mathrm{~K}^{-1}$ at about $870 \mathrm{~K}$ for the 2 at.\% Na-doped single crystal. Apparently, the lattice thermal conductivity of all $\mathrm{SnS}$ crystals had an intrinsically low value at elevated temperatures because of the strong lattice anharmonicity associated with the large Grüneisen parameters $\left(\gamma_{a}=3.9, \gamma_{b}=2.1, \gamma_{c}=2.3\right) .{ }^{[39]}$ We also observed comparably low lattice thermal conductivity values and large Grüneisen parameters for polycrystalline $\mathrm{SnS} .{ }^{[32]}$

Figure of merit: Figure 5(a) displays the temperature-dependent dimensionless TE figure of merit $(z T)$ for our $\mathrm{Sn}_{1-\mathrm{x}} \mathrm{Na}_{\mathrm{x}} \mathrm{S}$ single crystals measured along the $b$-axis direction. The $z T$ value of all $\mathrm{SnS}$ single crystals increased as the temperature rose in the entire temperature range, and the figure of merit also was enhanced by Na doping. The data in Figure 5(a) show 
that the highest $z T$ value of approximately 1.1 at $\sim 870 \mathrm{~K}$ was achieved for the $\mathrm{SnS}$ crystal with the Na content of $x=0.02$. This value was higher than that of Na-doped lead(II) sulfide $(\mathrm{PbS})$ with the same Na-doping content. ${ }^{[42]}$ As shown in Figures S3 and 5(b), we compared the highest $z T$ values and the average $z T$ values obtained with our SnS single crystals to corresponding values of several representative metal sulfides except superionic conductors as reported in the previous literature. Clearly, our Na-doped SnS single crystals are among the best TE sulfides in terms of peak $z T$ value as well as average $z T$. More important, the significant enhancement in the average $z T(\sim 180 \%)$, compared with the SnS polycrystalline material, makes the Na-doped SnS single crystal more plausible for practical TE applications. ${ }^{[45,46]}$ According to theoretical calculations, ${ }^{[39]}$ a higher $z T$ value $(\sim 1.9$ at $800 \mathrm{~K})$ for $\mathrm{SnS}$ single crystal samples along the $b$-axis direction could be realized by combining improved doping efficiencies for the higher PF with the reduced total thermal conductivity via forming solid solutions.

Anisotropy. According to the characteristic layered structure of $\mathrm{SnS}$ and the experimental results (see supporting information), we found that $\mathrm{SnS}$ single crystals exhibited strong textured character, indicating a strong anisotropy in the TE properties. ${ }^{[43,44]}$ Thus, all transport properties should be measured along the same direction (Figure S1(a)). Indeed, as shown in Figure 6, transport properties of pure $\mathrm{SnS}$ and $\mathrm{Na}_{0.02} \mathrm{Sn}_{0.98} \mathrm{~S}$ crystals displayed a strongly anisotropic character, with the electrical resistivity along the $a$-axis being much higher than that along the $b$-axis and the $c$-axis. With no obvious difference in the Seebeck coefficient measured at different directions, the PF along the $a$-axis clearly was less competitive than the other two directions. Additionally, the thermal conductivity along the $a$-axis for both $\mathrm{SnS}$ and $\mathrm{Na}_{0.02} \mathrm{Sn}_{0.98} \mathrm{~S}$ crystals was lower than the conductivity along the $b$-axis and the $c$-axis. This 
indicated a strongly anisotropic heat transport in the crystals, similar to that observed in SnSe. ${ }^{[26,27]}$

\section{Summary}

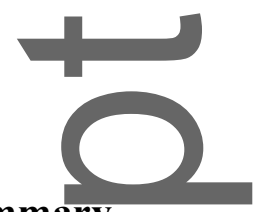

$$
\text { - }
$$

We prepared pristine and Na-doped SnS single crystals using a modified Bridgman method and studied their TE properties from room temperature to $873 \mathrm{~K}$. We observed strong anisotropy in TE transport properties and identified the best performance along the $b$-axis. The grain boundary-free nature of the crystals gave rise to their higher mobility as compared with their polycrystalline counterpart. We found that Na-doping significantly increased the concentration of holes, which, in turn, enhanced the electrical conductivity, leading to a much-improved PF from $0.19 \mathrm{~mW} \mathrm{~m}^{-1} \mathrm{~K}^{-2}$ for the pure $\mathrm{SnS}$ crystal to $2.0 \mathrm{~mW} \mathrm{~m} \mathrm{~K}^{-1} \mathrm{~K}^{-2}$ for the 2 at.\% Na-doped crystal. DFT calculations combined with the analysis based on the SPB model clearly verified the participation of the lower-lying heavy valence band in the transport process as a result of heavy $\mathrm{Na}$ doping. The participation of more than a single valence band benefited the Seebeck coefficient and the power factor. Strong anharmonicity of lattice vibrations and the highly anisotropic crystal structure of SnS maintained very low lattice thermal conductiyity. Of all the single crystalline $\mathrm{SnS}$ samples, the maximum $z T$ of 1.1 at 870 $\mathrm{K}$ was achieved in $\mathrm{Na}_{0.02} \mathrm{Sn}_{0.98} \mathrm{~S}$. This represented one of the highest $z T$ values obtained for a metal sulfide-based TE material. Our results demonstrated that $\mathrm{SnS}$ single crystals consisting of inexpensive, earth-abundant, and nontoxic elements attained high TE performance. Therefore, these crystals are potential TE materials for power generation applications.

\section{Experimental and theoretical methods}


Synthesis: We carefully weighed pure Sn granules (99.999\%), S powder (99.99\%), and Na chunks (99.9\%) according to the stoichiometry of $\mathrm{Sn}_{1-x} \mathrm{Na}_{x} \mathrm{~S}(x=0,0.001,0.01,0.02,0.03$, 0.04 ) and loaded the samples into cone-shaped quartz tubes under an argon atmosphere. We sealed the tubes under $\sim 5 \times 10^{-4} \mathrm{~Pa}$ and placed them in another (larger diameter) quartz tube, which we again evacuated and flame-sealed. We did this to protect the samples from oxidation as the cone-shaped quartz tube breaks easily when the crystal structure of SnS undergoes a phase transition from the Cmcm to the Pnma phase upon cooling. We slowly heated the tubes up to $1273 \mathrm{~K}$ over $20 \mathrm{~h}$ and soaked the tubes at that temperature for $15 \mathrm{~h}$. Subsequently, we cooled the tubes to $1100 \mathrm{~K}$ as they were lowered at a rate of $1.5 \mathrm{~mm} \mathrm{~h}^{-1}$. This carefully monitored process resulted in $\mathrm{Sn}_{1-\mathrm{x}} \mathrm{Na}_{\mathrm{x}} \mathrm{S}$ single crystal ingots with a diameter of $14 \mathrm{~mm}$ and a height of $45 \mathrm{~mm} .^{[47]}$

Measurements: We cut the obtained single crystal ingots into $\sim 8.5 \mathrm{~mm} \times 2 \mathrm{~mm} \times 2 \mathrm{~mm}$ bars for electrical property measurements and square-shaped samples with dimensions of $\sim 6$ $\mathrm{mm} \times 6 \mathrm{~mm} \times 1.5 \mathrm{~mm}$ to take thermal conductivity measurements along the three directions (along the $a$-, $b$ - and $c$-axis, respectively), as shown in Figure S1(a). We performed the electronic transport property measurements on a commercial system (LSR-3, Linseis) under a static helium atmosphere. We measured the temperature-dependent Hall coefficient $\left(R_{\mathrm{H}}\right)$ using a homemade apparatus. We measured thermal diffusivity $(D)$ using the LFA 457 (Netzsch, Selb, Germany) instrument and obtained the heat capacity $\left(C_{p}\right)$ through differential scanning calorimetry (DSC; 404 F3, Netzsch), as shown in Figure S2. We calculated thermal conductivity $(\kappa)$ using $\kappa=\rho D C_{p}$, where $\rho$ is the density (see Table S3) measured by the Archimedes' method on a commercial instrument (BR-120N, Beyongtest). The estimated error in electricalresistivity, Seebeck coefficient, and thermal conductivity were about $5 \%$, 
$7 \%$, and $5 \%$, respectively; and we estimated the overall uncertainty of $z T$ at around $15 \%$ (error bars are displayed in the relevant figures). We determined the crystal structures using PANalytical X'pert apparatus (Netherlands) with $\mathrm{CuK} \alpha$ radiation. We performed STEM and EDS mappings at $300 \mathrm{kV}$ on a probe-corrected FEI Titan G2 60-300 microscope (Hillsboro, OR, USA) with a super-X system. The band gap was analyzed by ultraviolet-visible non-infrared spectrophotometer (UV-3600, Shimadzu, Kyoto, Japan).

DFT calculations: The electronic band structure was computed within the framework of DFT using the Vienna ab-initio Simulation Package, ${ }^{[36]}$ in which we used the projector augmented plane wave $(\mathrm{PAW})^{[37]}$ method for structure relaxation. We defined the exchange-correlation function using a generalized gradient approximation (GGA) of Perdew-Burke-Ernzerhof. ${ }^{[38]}$ The total energies were numerically converged to $1 \times 10^{-6} \mathrm{eV}$ using a basis set energy cutoff of $500 \mathrm{eV}$. We relaxed the atomic positions until the forces on the atoms were smaller than $0.01 \mathrm{eV} \AA^{-1}$. For the Brillouin zone integrations, we used the Monkhorst-Pack $k$-mesh scheme with $3 \times 9 \times 9$ for the cell system.

\section{Supporting Information}

Supporting Information is available from the Wiley Online Library or from the author.

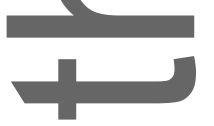

\section{Acknowledgements}

This work was financially supported in part by the National Natural Science Foundation of China (Grant Nos. 11674040, 11604032, 51472036, 51672270), the Fundamental Research 
Funds for the Central Universities (106112016CDJZR308808). The work conducted at the

Chongqing Institute of Green and Intelligent Technology, Chinese Academy of Sciences is also supported by Key Research Program of Frontier Sciences, CAS, Grant No.

QYZDB-SSW-SLH016 and the Project for Fundamental and Frontier Research in Chongqing (CSTC2015JCYJBX0026).

\section{References}

[1] L. E.Bell, Seience 2008, 321, 1457-1461.

[2] M. S. Dresselhaus, G. Chen, M. Y. Tang, R. G. Yang, H. Lee, D. Z. Wang, Z. F. Ren, J. P. Fleurial, P. Gogna, Adv. Mater. 2007, 19, 1043-1053.

[3] J. P. Heremans, M. S. Dresselhaus, L. E. Bell, D. T. Morelli, Nat. Nanotechnol. 2013, 8, $471-473$.

[4] X. Lu, D. T. Morelli, Y. Wang, W. Lai, Y. Xia, V. Ozolins, Chem. Mater. 2016, 28, $1781-1786$.

[5] Q. Tan, J. F. Li, J. Electron. Mater. 2014, 43, 2435-2439.

[6] G. J. Snyder, E. S. Toberer, Nat. Mater. 2008, 7, 105-114.

[7] T.Zhu, Y. Liu, C. Fu, J. P. Heremans, J. G. Snyder, X. Zhao, Adv. Mater. 2017, 29, 1605884.

[8] M. Zhou, G. J. Snyder, L. Li, L. D. Zhao, Inorg. Chem. Front. 2016, 3, 1449-1463. 
[9] K. F. Hsu, S. Loo, F. Guo, W. Chen, J. S. Dyck, C. Uher, T. Hogan, E. K.

Polychroniadis, M. G. Kanatzidis, Science 2004, 303, 818-821.

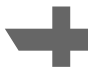

[10] J. P. Heremans, C. M. Thrush, D. T. Morelli, Phys. Rev. B 2004, 70, 115334.

[11] O. Falkenbach, J. Tinz, A. S. Schulze, E. Mueller, S. Schlecht, Phys. Status Solidi A 2016, 213, 699-705.

[12] Z. W. Chen, B. H. Ge, W. Li, S. Q. Lin, J. W. Shen, Y. J. Chang, R. Hanus, G. J.

Snyder, Y. Z.Pei, Nat. Commun. 2017, 8, 13828.

[13] S. I. Kim, K. H. Lee, H. A. Mun, H. S. Kim, S. W. Hwang, J. W. Roh, D. J. Yang, W. H. Shin, X. S. Li, Y. H. Lee, Science, 2015, 348, 109-114.

[14] J. D. Wasscher, W. Albers, C. Haas, Solid-State Electron.1963, 6, 261-264.

[15] S. Q. Lin, W. Li, X. Y. Zhang, J. Li, Z. W. Chen, Y. Z. Pei, Inorg. Chem. Front. 2017, $4,1066-1072$

[16] Y. Pei, L. Zheng, W. Li, S. Lin, Z. Chen, Y. Wang, X. Xu, H. Yu, Y. Chen, B. Ge, Adv. Electron. Mater. 2016, 2, 1600019.

[17] W. Yao, D. Yang, Y. Yan, K. Peng, H. Zhan, A. Liu, X. Lu, G. Wang, X. Zhou, ACS Appl. mater. Interfaces 2017, 9, 10595-10601.

[18] M. D. Nielsen, V. Ozolins, J. P. Heremans, Energ. Environ. Sci. 2013, 6, 570-578.

[19] D. Yang, W. Yao, Y. Yan, W. Qiu, L. Guo, X. Lu, C. Uher, X. Han, G. Wang, T. Yang, X.Zhou, NPG Asia Mater. 2017, 9, e387. 
[20] D. Yang, W. Yao, Q. Chen, K. Peng, P. Jiang, X. Lu, C.Uher, T. Yang, G. Wang, X. Zhou, Chem. Mater. 2016, 28 (6), 1611-1615.

r

[21] Y. Z. Pei, A. D. LaLonde, N. A. Heinz, X. Y. Shi, S. Iwanaga, H. Wang, L. D. Chen,

G. J. Snyder, Adv. Mater. 2011, 23, 5674-5678.

[22] S. Bhattacharya, N. S. Gunda, R. Stern, S. Jacobs, R. Chmielowski, G. Dennler, G. K. H. Madsen, Phys. Chem. Chem. Phys. 2015, 17, 9161-9166.

[23] Y. Z.Pei, X. Y. Shi, A. LaLonde, H. Wang, L. D. Chen, G. J. Snyder, Nature 2011, $473,66-69$.

[24] X. La, W. Yao, G. Wang, X. Zhou, D. Morelli, Y. Zhang, H. Chi, S. Hui, C. Uher, J. Mater. Chem. A 2016, 4, 17096-17103.

[25] J.Yang, L. L. Xi, W. J. Qiu, L. H. Wu, X Shi, L. D. Chen, J. H. Yang, W. Q. Zhang, C. Uher, D. J. Singh, npj Comp. Mater. 2016, 2, 15015.

[26] L. D. Zhao, S. H. Lo, Y. Zhang, H. Sun, G. Tan, C. Uher, C. Wolverton, V. P. Dravid, M. G. Kanatzidis, Nature 2014, 508, 373-7.

[27] K. Peng, X. Lu, H. Zhan, S. Hui, X. Tang, G. Wang, J. Dai, C. Uher, G. Wang, X. Zhou, Energ. Environ. Sci. 2016, 9, 454-460.

[28] T. Duong, V. Q. Nguyen, G. Duvjir, V. T. Duong, S. Kwon, J. Y. Song, J. K. Lee, J. E. Lee, S. Park, T. Min, J. Lee, J. Kim, S. Cho, Nat. Commun. 2016, 7, 13713.

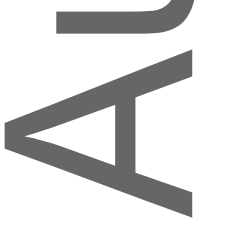


[29] L. D. Zhao, G. J. Tan, S. Q. Hao, J. Q. He, Y. L. Pei, H. Chi, H. Wang, S. K. Gong, H.

B. Xu, V. P. Dravid, C. Uher, G. J. Snyder, C. Wolverton, M. G. Kanatzidis, Science 2016, [30] K. L. Peng, B. Zhang, H Wu, X. L. Cao, A. Li, D. F. Yang, X. Lu, G. Y. Wang, X. D. Han, C. Uber,X. Y. Zhou, Mater. Today, DOI: 10.1016/j.mattod.2017.11.005.

[31] J. S. Rhyee, K. H. Lee, S. M. Lee, E. Cho, S. I. Kim, E. Lee, Y. S. Kwon, J. H. Shim, G. Kotliar, Nature 2009, 459, 965-8.

[32] R. Guo, X. Wang, Y. Kuang, B. Huang, Phys. Rev. B 2015, 92, 115202.

[33] B. Z. Sun, Z. Ma, C. He, K. Wu, RSC Adv. 2015, 5, 56382-56390.

[34] B. Zhou, S. Li, W. Li, J. Li, X. Zhang, S. Lin, Z. Chen, Y. Pei, ACS Appl. mater. Interfaces 2017,9, 34033-34041.

[35] Q. Tan, E. D. Zhao, J. F. Li, C. F. Wu, T. R. Wei, Z. B. Xing, M. G. Kanatzidis, J. Mater. Chem. A 2014, 2, 17302-17306.

[36] G. Kresse, J. Furthmuller, Phys. Rev. B 1996, 54, 11169-11186.

[37] G. Kresse, D. Joubert, Phys. Rev. B 1999, 59, 1758-1775.

[38] J.P. Perdew, K. Burke, M. Ernzerhof, Phys. Rev. Lett. 1996, 77, 3865-3868.

[39] S. Hao, V.P. Dravid, M. G. Kanatzidis, C. Wolverton, APL Mater. 2016, 4, 104505.

[40] G. K. H. Madsen, D. J. Singh Phys. Commun. 2006, 175,67-71.

[41] G. Ding, G. Gao, K. Yao, Sci. Rep. 2015, 5, 9567. 
[42] L. D. Zhao, J. He, S. Hao, C. I. Wu, T. P. Hogan, C. Wolverton, V. P. Dravid, M. G. Kanatzidis, J. Am. Chem. Soc. 2012, 134, 16327-36.

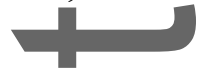

[43] Asfandiyar, T. R. Wei, Z. Li, F. H. Sun, Y. Pan, C. F. Wu, M. U. Farooq, H. Tang, F.

Li, B. Li, J. F. Li, Sci. Rep. 2017, 7, 43262.

[44] M. M. Nassary, J. Alloys Compd. 2005, 398, 21-25.

[45] G. Tan, L. D. Zhao, M. G. Kanatzidis, Chem. Rev. 2016, 116, 12123-12149.

\section{P}

[46] L. Yang, Z. G. Chen, M. S. Dargusch, J. Zou, Adv. Electron. Mater. 2017, 1701797.

[47] W. Albers, C. Haas, F. van der Maesen, J. Phys. Chem. Solids, 1960, 15, 306-310.

[48] K. E. Peng, H. Wu, Y. C. Yan, L. J. Guo, G. Y. Wang, X. Lu, X. Y. Zhou, J. Mater. Chem. A 2017, 5, 14053-14060.

[49] X. Lu, D. T. Morelli, Y. Xia, F. Zhou, V. Ozolins, H. Chi, X. Zhou, C. Uher, Adv. Energy Mater. 2013, 3, 342-348.

[50] Y. Yang, P. Ying, J. Wang, X. Liu, Z. Du, Y. Chao, J. Cui, J. Mater. Chem. A 2017, 5, $18808-1881$

[51] H. W. Zhae, X. X. Xu, C. Li, R. M Tian, R. Z. Zhang, R. Huang, Y. Lu, D. X. Li, X.

H. Hu, L.Pan, Y.F. Wang, J. Mater. Chem. A 2017, 5, 23267-23275

[52] G. Tan, S. Hao, J. Zhao, C. Wolverton, M. G. Kanatzidis, J. Am. Chem. Soc. 2017, $139,6467-6473$.

[53] K. Biswas, L. D. Zhao, M. G. Kanatzidis, Adv. Energy Mater. 2012, 2, 634-638. 
[54] E. Guilmeau, Y. Bréard, A. Maignan, Appl. Phys. Lett. 2011, 99, 052107.

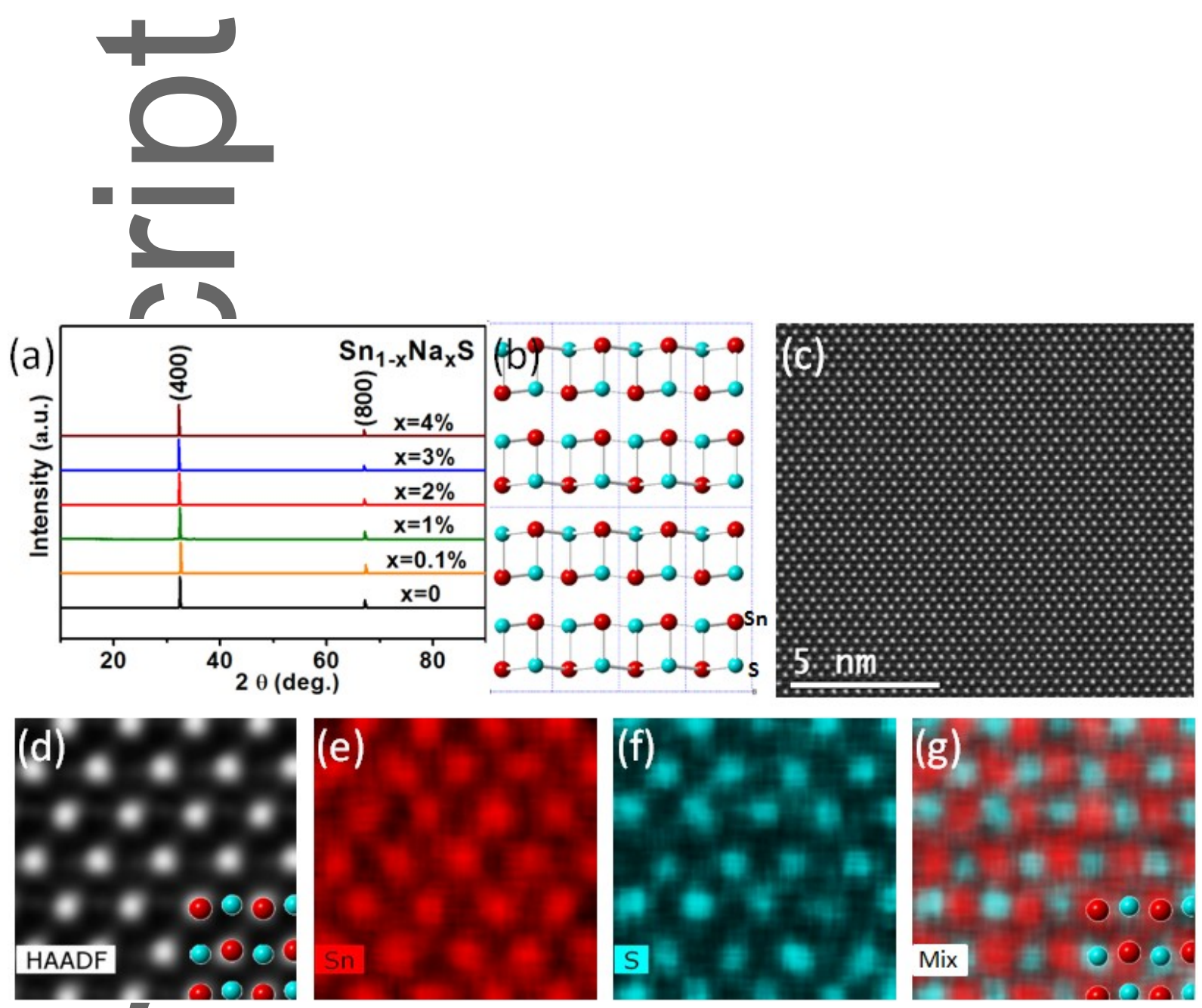

Figure 1. Structural characterization of $\mathrm{Sn}_{1-x} \mathrm{Na}_{x} \mathrm{~S}$ single crystals. (a) XRD patterns. (b) The atomic arrangement of SnS (Pnma) projected along the [001] direction. (c) The [001] HAADF image of $\mathrm{Sn}_{0.98} \mathrm{Na}_{0.02} \mathrm{~S}$. (d)-(g) STEM-EDS mapping of $\mathrm{Sn}_{0.98} \mathrm{Na}_{0.02} \mathrm{~S}$ with the HAADF image and the corresponding elemental mappings. A structure schematic is attached at (d) the bottom right corner of the HAADF image and at $(\mathrm{g})$ the mixture mapping.

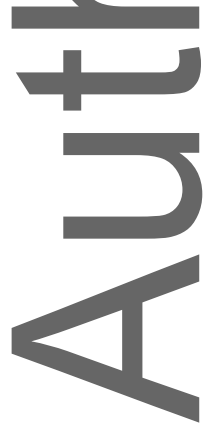



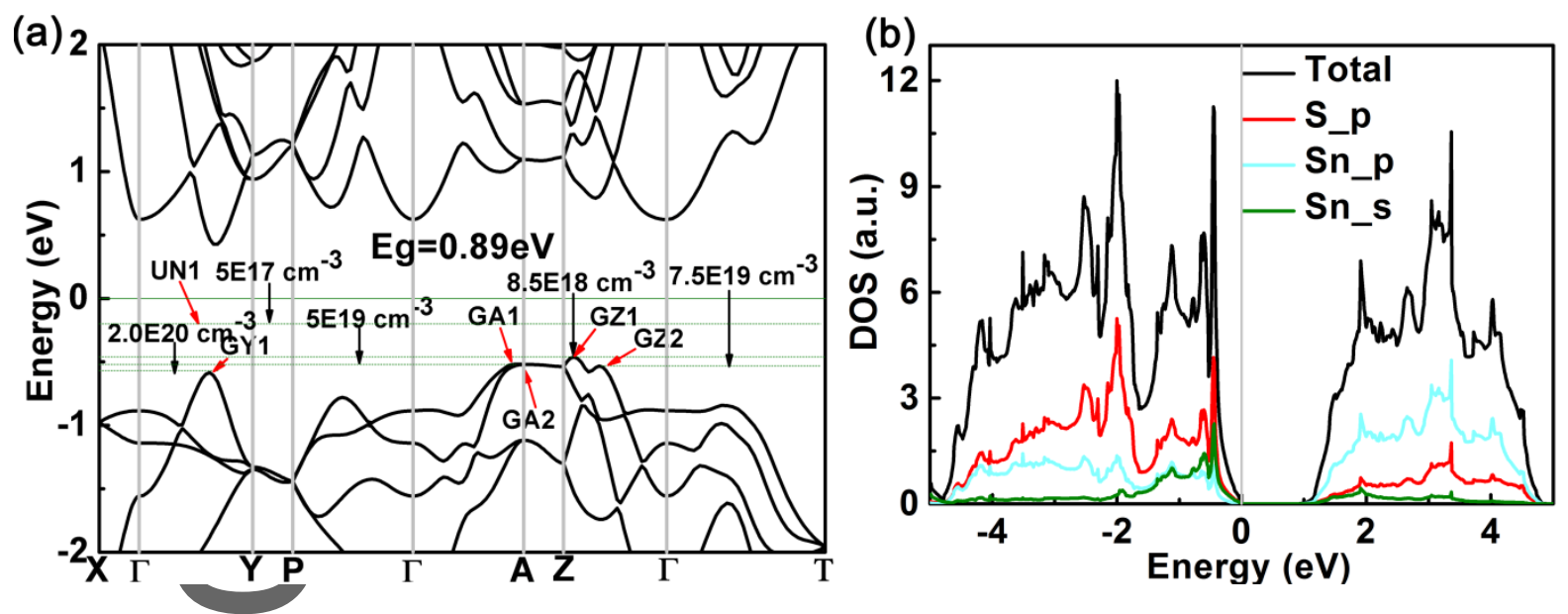

Figure 2. (a) The calculated electronic band structure of $\mathrm{SnS}$ and carrier density as a function of the Fermilevel. (b) The total and partial density of states for the pristine SnS.
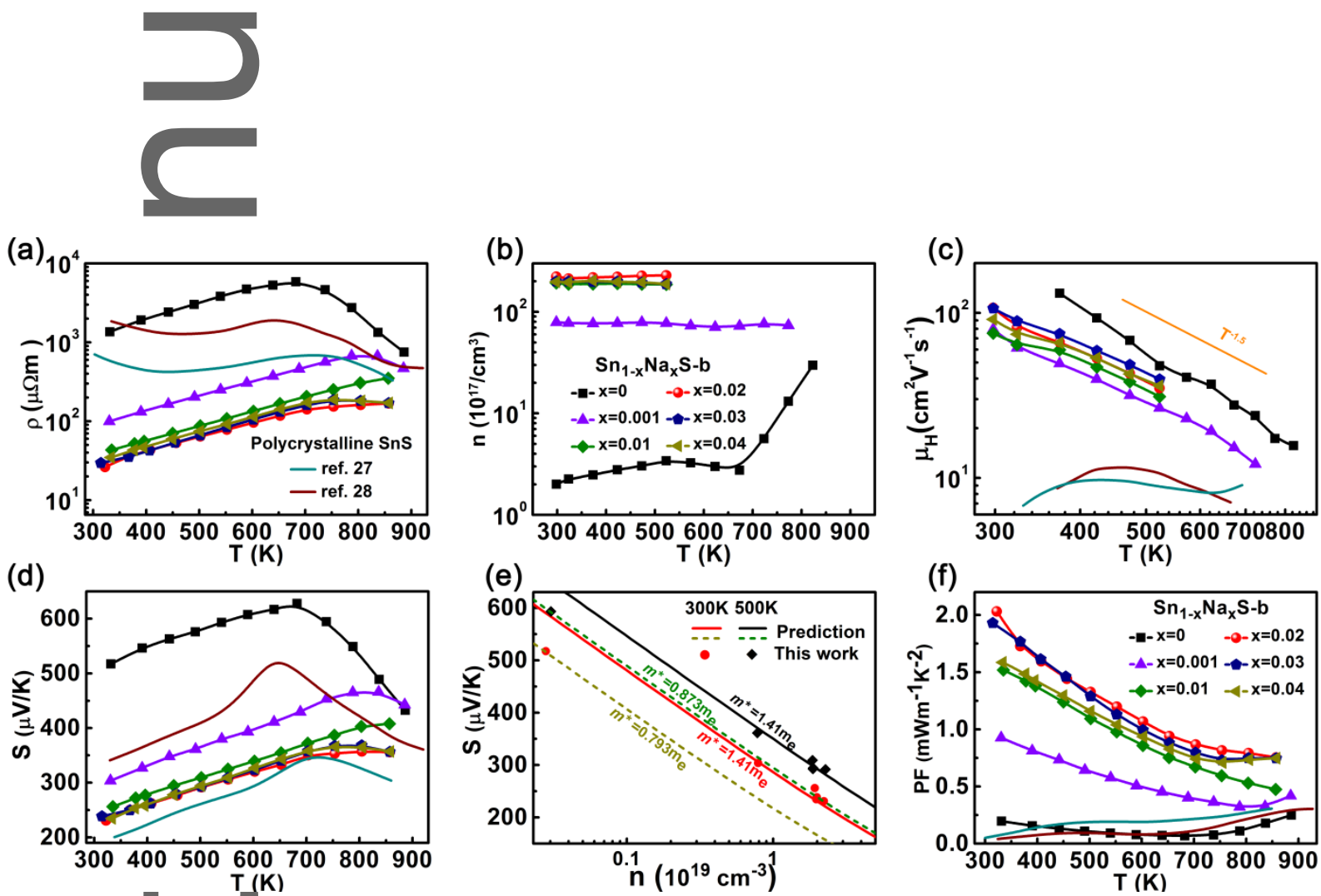

Figure 3. Temperature-dependent electronic properties of $\operatorname{Sn}_{1-x} \mathrm{Na}_{x} \mathrm{~S}(x=0,0.001,0.01,0.02$, 0.03 , and 0.04) single crystals along the crystallographic $b$-axis: (a) electrical resistivity; (b) Hall carrier coneentration; (c) Hall mobility; (d) Seebeck coefficient; (e) Pisarenko plot; and (f) power factor. Data on the temperature-dependent transport properties reported for polycrystalline $\mathrm{SnS}$ doped with $\mathrm{Na}^{[34]}$ and $\mathrm{Ag}^{[35]}$ are shown for comparison. 

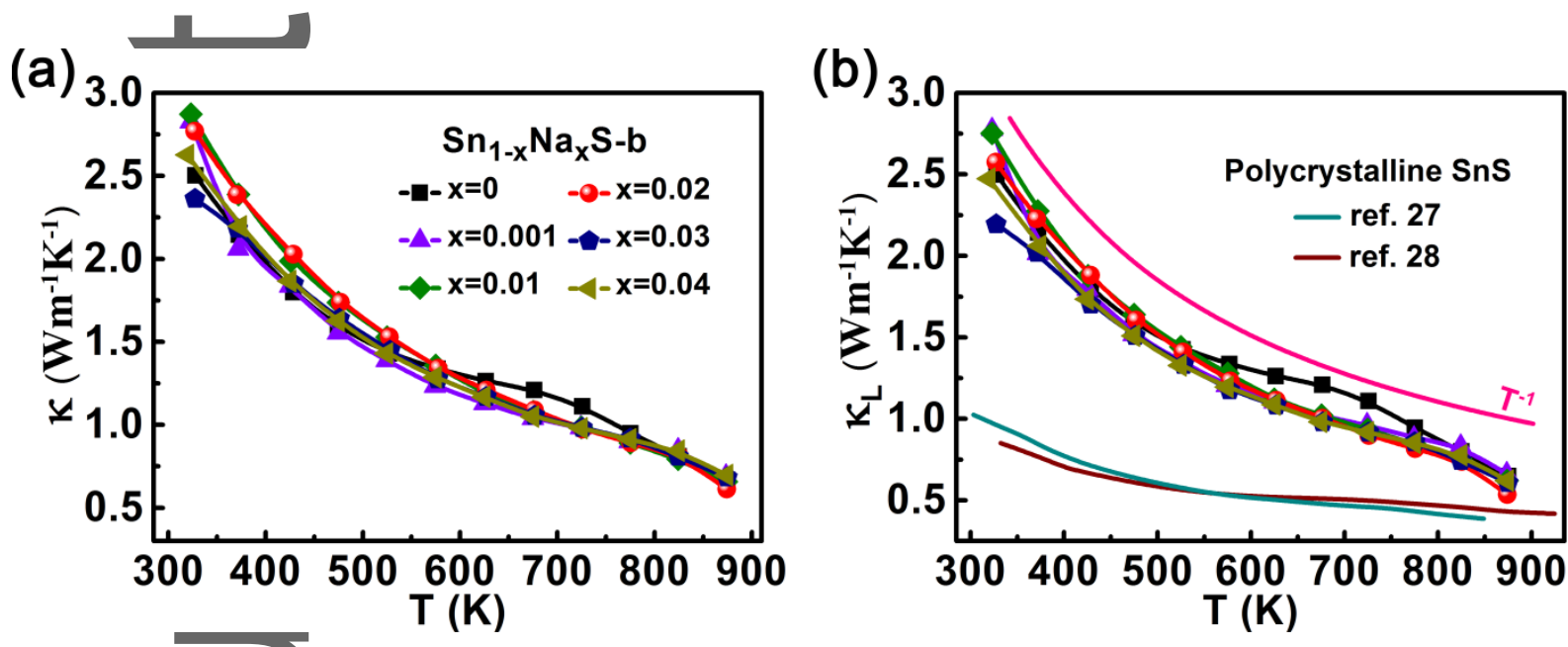

Figure 4. Temperature-dependent thermal transport properties of $\mathrm{Sn}_{1-x} \mathrm{Na}_{x} \mathrm{~S}(x=0,0.001$, $0.01,0.02,0.03$, and 0.04 ) single crystals along the crystallographic $b$-axis: (a) the total thermal conductivity; and (b) the lattice thermal conductivity.
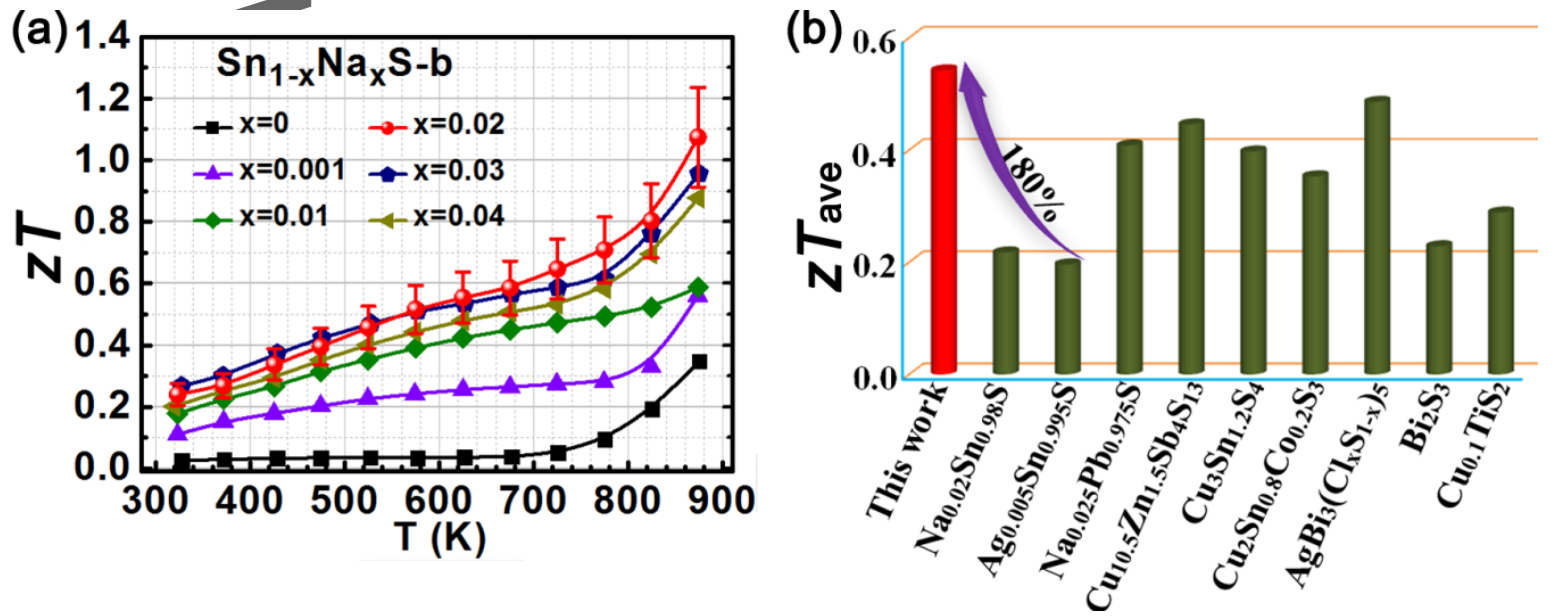

Figure 5. (a) $z T$ yalues for $\mathrm{Sn}_{1-x} \mathrm{Na}_{x} \mathrm{~S}(x=0,0.001,0.01,0.02,0.03$ and 0.04$)$ single crystals along the crystallographic $b$-axis. (b) The average value of $z T$ for metal sulfides ${ }^{[34,35,42,49-54]}$ in the temperatures range from $\sim 300$ to $\sim 900 \mathrm{~K}$. The detail methods ${ }^{[48]}$ are displayed in supporting information. 
(a)
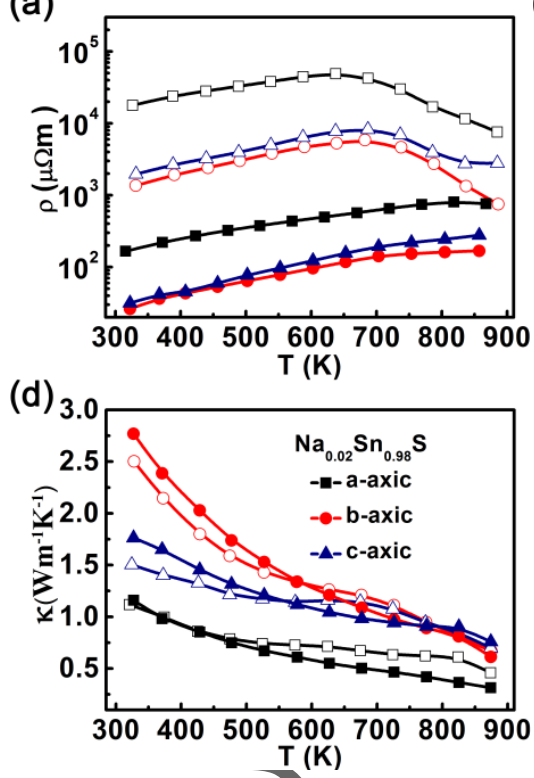

(b)

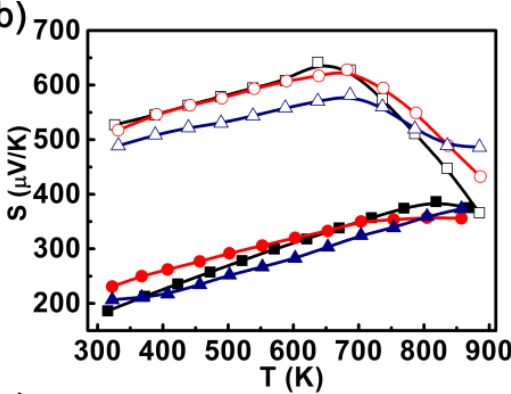

(e)

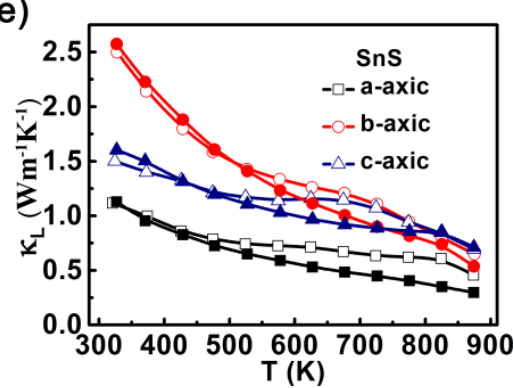

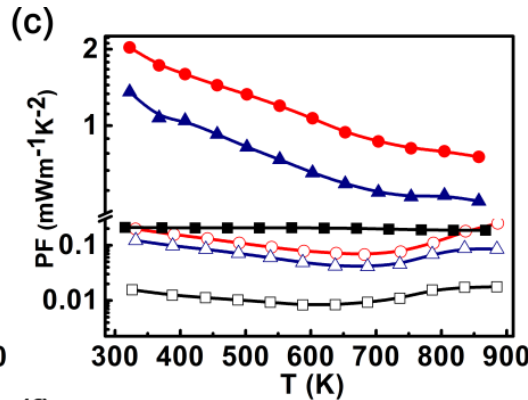

(f) 1

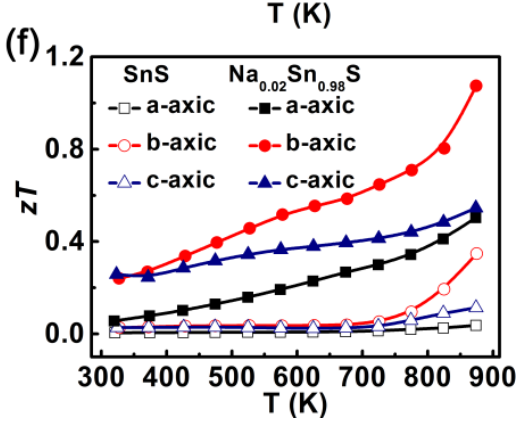

Figure 6. The anisotropic thermoelectric properties of $\mathrm{Na}_{0.02} \mathrm{Sn}_{0.98} \mathrm{~S}$ and $\mathrm{SnS}$ single crystals.

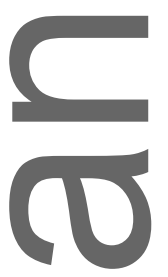

Table 1. Effective masses of $\mathrm{SnS}$ obtained by fitting the band structure.

\begin{tabular}{|c|c|c|c|c|c|c|c|}
\hline $\begin{array}{c}\text { Vatence } \\
\text { band tops } \\
(\mathrm{eV})\end{array}$ & $\mathrm{VBM}$ & $m_{k x}\left(m_{e}\right)$ & $m_{k y}\left(m_{e}\right)$ & $m_{k z}\left(m_{e}\right)$ & $\begin{array}{c}m_{\mathrm{c}}{ }^{*} \\
\left.m_{e}\right)\end{array}$ & $\begin{array}{c}m_{b}{ }^{*}( \\
\left.m_{e}\right)\end{array}$ & $\begin{array}{c}m_{d}{ }^{*} \\
\left.m_{e}\right)\end{array}$ \\
\hline $\mathrm{GZ1}$ & 0.0 & 3.051 & 0.338 & 0.222 & 0.385 & 0.612 & 0.971 \\
\hline $\mathrm{GA}$ & 0.055 & 6.781 & 0.740 & 0.358 & 0.698 & 1.215 & 3.063 \\
\hline $\mathrm{GA} 2$ & 0.059 & 20.01 & 6.270 & 0.415 & 1.145 & 3.734 & 9.409 \\
\hline $\mathrm{GZ2}$ & 0.071 & 1.278 & 0.798 & 0.233 & 0.474 & 0.619 & 0.983 \\
\hline $\mathrm{GY} 1$ & 0.123 & 0.319 & 0.291 & 0.169 & 0.240 & 0.251 & 0.397 \\
\hline
\end{tabular}


Table 2. Room temperature carrier concentration obtained by different methods.

\begin{tabular}{|c|c|c|c|c|c|}
\hline$\frac{\mathrm{F}}{\mathrm{p}}$ & $\begin{array}{c}\text { Fermi level } \\
\qquad(\mathrm{eV})\end{array}$ & $\begin{array}{l}\text { Calculation } \\
\qquad\left(\mathrm{cm}^{-3}\right)\end{array}$ & $\begin{array}{l}\text { Samples } \\
\mathrm{Na}_{x} \mathrm{Sn}_{1-x} \mathrm{~S}\end{array}$ & $\begin{array}{c}\text { SPB model } \\
\left(\mathrm{cm}^{-3}\right)\end{array}$ & $\begin{array}{c}\text { Hall carrier } \\
\text { concentration } \\
\left(\mathrm{cm}^{-3}\right)\end{array}$ \\
\hline UN1 & -0.26 & $5.0 \mathrm{E} 17$ & $x=0$ & $3.7 \mathrm{E} 17$ & $2.0 \mathrm{E} 17$ \\
\hline \multirow{5}{*}{ GZ1 } & \multirow{5}{*}{0.0} & \multirow{5}{*}{$8.5 \mathrm{E} 18$} & $x=0.001$ & $8.1 \mathrm{E} 18$ & $7.8 \mathrm{E} 18$ \\
\hline & & & $x=0.01$ & 2.0E19 & $1.9 \mathrm{E} 19$ \\
\hline & & & $x=0.02$ & $2.6 \mathrm{E} 19$ & 2.3E19 \\
\hline & & & $x=0.03$ & $2.4 \mathrm{E} 19$ & $2.0 \mathrm{E} 19$ \\
\hline & & & $x=0.04$ & $2.5 \mathrm{E} 19$ & 1.9E19 \\
\hline GA1 & 0.055 & $5.0 \mathrm{E} 19$ & & & \\
\hline
\end{tabular}

\section{TOC}

Large size $\mathrm{Sn}_{1-\mathrm{x}} \mathrm{Na}_{\mathrm{x}} \mathrm{S}$ single crystals were firstly obtained using a modified Bridgman method. The multiple band feature along with the single crystalline nature favors the large power factor, finally leading to the highest $z T$ value of $\sim 1.1$ at $870 \mathrm{~K}$ and the average $z T$ of $\sim 0.54$ from $300 \mathrm{~K}$ to $870 \mathrm{~K}$ for 2 at.\% Na-doped $\mathrm{SnS}$ single crystal along the $b$-axis direction, which is one of the best TE sulfides.
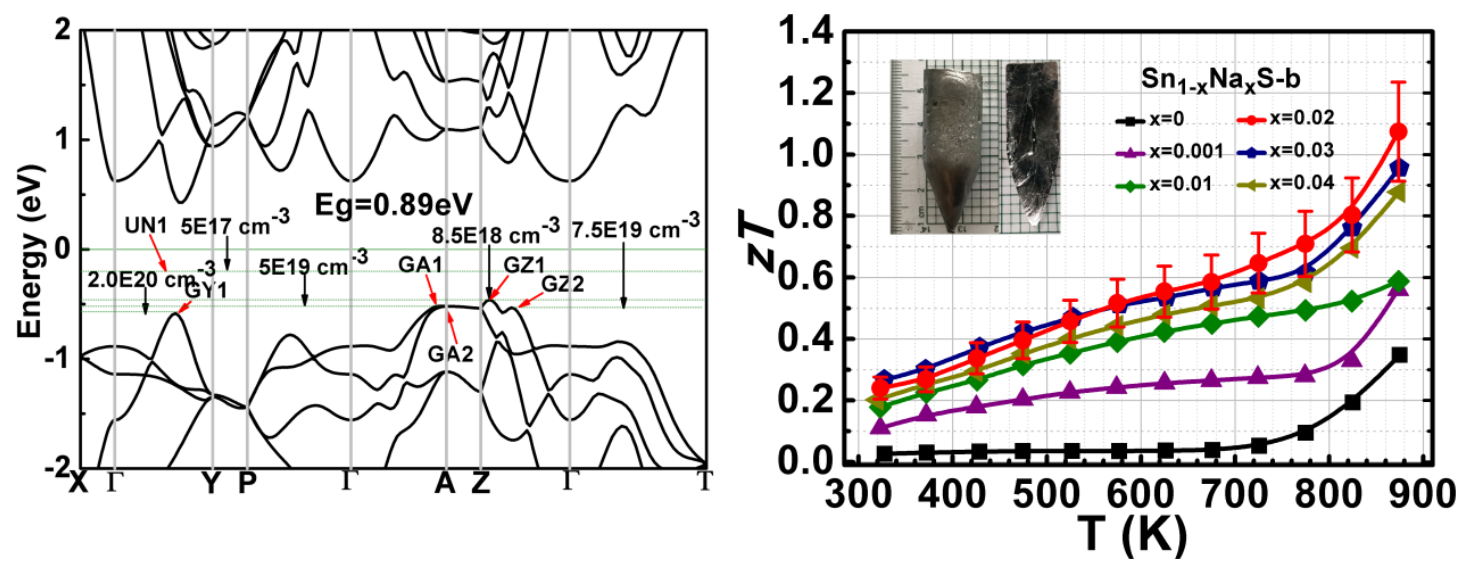